[Original publication information:

RECEIVED 31 MAY; ACCEPTED 12 OCTOBER; PUBLISHED ONLINE 26

NOVEMBER 2006; DOI:10.1038/NMETH975

NATURE METHODS, VOL.4 NO.1, JANUARY 2007, pages 39 - 42]

[Figures are not reproduced]

[Begin of page 39]

\title{
Fast manipulation of cellular cAMP level by light in vivo
}

Saskia Schröder-Lang ${ }^{1}$, Martin Schwärzel ${ }^{2}$, Reinhard Seifert ${ }^{3}$, Timo Strünker ${ }^{3}$, Suneel Kateriya ${ }^{4}$, Jens Looser ${ }^{5}$, Masakatsu Watanabe ${ }^{6,7}$, U Benjamin Kaupp ${ }^{3}$, Peter Hegemann ${ }^{4} \&$ Georg Nagel ${ }^{1,5}$

${ }^{1}$ Max-Planck-Institut für Biophysik, 60439 Frankfurt, Germany. ${ }^{2}$ Saarland Universität, Naturwissenschaftlich-Technische Fakultät III, FR 8.3, Zoologie \& Physiologie, 66041 Saarbrücken, Germany. ${ }^{3}$ Forschungszentrum Jülich, Institut für Neurowissenschaften und Biophysik, Abteilung Zelluläre Signalverarbeitung, INB-1, 52425 Jülich, Germany. ${ }^{4}$ Humboldt Universität zu Berlin, Experimentelle Biophysik, 10115 Berlin, Germany. ${ }^{5}$ Universität Würzburg, Julius-von-Sachs-Institut für Biowissenschaften, 97082 Würzburg, Germany. ${ }^{6}$ National Institute for Basic Biology, Okazaki, 444-8585, Japan.

${ }^{7}$ School of Advanced Sciences, Graduate University of Advanced Studies (SOKENDAI), Hayama, 240-0193, Japan. Correspondence should be addressed to G.N. (nagel@botanik.uni-wuerzburg.de) or M.S.

(m.schwaerzel@mx.uni-saarland.de).

Abstract:

The flagellate Euglena gracilis contains a photoactivated adenylyl cyclase (PAC), consisting of the flavoproteins PACa and PACb. Here we report functional expression of PACs in Xenopus laevis oocytes, HEK293 cells and in Drosophila melanogaster, where neuronal expression yields light-induced changes in behavior. The activity of PACs is strongly and reversibly enhanced by blue light, providing a powerful tool for light-induced manipulation of cAMP in animal cells.

cAMP is a ubiquitous second messenger across phyla ${ }^{1}$ and multiple adenylyl cyclases, and phosphodiesterases are involved in its formation and degradation, respectively. A light-activated adenylyl cyclase that is crucial for photoavoidance has been identified in the unicellular flagellate Euglena gracilis ${ }^{2}$. This adenylyl cyclase is composed of two PACa and two PACb subunits, which exhibit adenylyl cyclase activity that is enhanced by blue light. Each subunit harbors two BLUF-type photoreceptor domains, binding flavin adenine dinucleotide ${ }^{3,4}$, and two catalytic domains that are homologous to 
class III adenylyl cyclases ${ }^{2}$. Until now, it was unclear whether the individual subunits are catalytically active and whether they are active in animal cells. Manipulation of cellular signaling in live animals with the help of genetically encoded light-sensitive proteins has become feasible in recent years ${ }^{5-7}$. Expression of a light-sensitive adenylyl cyclase in cells would allow the manipulation of cAMP with exquisite spatiotemporal control. To this end, we functionally expressed PACs (encoded by PACa and PACb) in two popular expression systems, X. laevis oocytes and HEK293 cells. Moreover, transgenic D. melanogaster flies demonstrated functional PAC expression by showing blue light-induced behavioral changes.

We expressed c-myc-tagged PACa and PACb in Xenopus oocytes and detected products of $\mathrm{B} 110 \mathrm{kDa}$ and $\mathrm{B} 90 \mathrm{kDa}$ in PACa-and

$\mathrm{PACb}$-expressing oocytes, in good agreement with the respective molecular weight of PACa (112 kDa) and PACb (94 kDa; Supplementary Fig. 1 online and Supplementary Methods online). We determined the total intracellular concentration of cAMP ([cAMP]i) in single oocytes by an immunoassay (Fig. 1a and Supplementary Data online). Control oocytes had a mean [cAMP]i of $1.3 \pm 0.6 \mathrm{mM}$. PACa-expressing oocytes displayed a pronounced adenylylcyclase activity at rest. After $4 \mathrm{~d}$ of expression in the dark, [cAMP]i was B20fold enhanced compared to controls. When we irradiated PACa-expressing oocytes by blue light for $5 \mathrm{~min}$, [cAMP]i increased further about tenfold, demonstrating light-stimulated adenylyl cyclase activity. We emphasize that the high [cAMP]i values obtained with the immunoassay refer to total [cAMP]i (much of it bound to cAMP-binding proteins). Control and PACb-expressing oocytes displayed no substantial changes in [cAMP]i upon blue-light irradiation for $5 \mathrm{~min}$ (Fig. 1a and Supplementary Data). The [cAMP]i of PACbexpressing oocytes was not substantially different from that of control oocytes. We used the human cystic fibrosis transmembrane conductance regulator (CFTR) as a cAMP sensor to monitor light-induced changes in [cAMP]i.CFTR isa $\mathrm{Cl}^{-}$channel that is activated by phosphorylation via cAMP-dependent protein kinase (PKA) ${ }^{9}$.We coexpressed CFTR with PACa,PACb,or PACa and $\mathrm{PACb}$ in oocytes. The high basal activity of PACa required us to reduce the expression level by injecting only B200 pg of cRNA. Application of the phosphodiesterase inhibitor, IBMX, and the activator of endogenous adenylyl cyclase, forskolin, caused a large increase in membrane conductance (Fig. 1b), as has been previously shown for cells that express CFTR only ${ }^{10}$. A short pulse of blue light mimicked this pharmacological effect by causing a similar increase in conductance after a delay of $15^{-20} \mathrm{~s}$ (Fig. 1b). The amplitude of the response increased with the intensity or duration of light stimulation (Supplementary Fig. 2 online). For strong irradiation, the delay of the electrical response was as short as $2 \mathrm{~s}$ (Fig. 1c).

When we injected B200 pg of PACb cRNA into oocytes, we observed no lightinduced change in conductance. When we injected 20 ng of PACb cRNA, however, irradiation of oocytes with blue light caused an increase in conductance (Fig. 1b)similar to that of oocytes injected with $200 \mathrm{pg}$ of PACa cRNA. The results of experiments in which PACs were coexpressed with CFTR in oocytes are summarized in Figure 1d.

To examine the kinetics of the light-induced cAMP production, we coexpressed PACa or PACb with cyclic nucleotide-gated (CNG) channels. CNG channels are directly opened by cAMP and cGMP, and mutant CNG channels with different sensitivities are available. For the study of PACa, we 
selected the mutant CNGA2-T537S

[Begin of page 40 ]

(Bos taurus CNGA2), which is characterized by a constant of half-maximal activation, KcAMP, of 14 mM (ref. 11). Coexpression of PACa and CNGA2$\mathrm{T} 537 \mathrm{~S}$ gave rise to a fast and reversible lightinduced increase of conductance (Fig. 1e). The light-induced activity of PACb was not sufficient to activate the CNGA2-T537S channel (data not shown). Therefore, we coexpressed PACb with a more sensitive mutant CNGA2-C46oW,E583M (KcAMP 1/4 $1 \mathrm{mM}$; ref. 12; Rattus norvegicus CNGA2). Oocytes, expressing PACb and CNGA2C460W,E583M, showed a fast and reversible light response (Fig. 1e). We also expressed PACa with the CNGA2C460W,E583M channel to estimate the rate of PACa activation. The photocurrent increased almost instantaneously after the onset of irradiation (Fig. 1f). We estimate the time constant for the activation of PACa to be $\mathrm{r} 2 \mathrm{O} \mathrm{ms}$, that is, the time resolution of the recordings. We also found that light-dependent activity of PACa switches off within a few milliseconds after light has been switched off (Supplementary Fig. 2), confirming and extending results on purified PACa-PACb ${ }^{13}$. It was not possible to switch off PAC by applying light of different wavelengths to specifically excite a photocycle intermediate. This agrees with a minimal spectral shift (10 $\mathrm{nm}$ to the red) of the BLUF signaling state ${ }^{3,4}$.

To examine the suitability of PACs as a tool to manipulate [cAMP]i, we expressed PACa in HEK293 cells with CNGA2T537M as a cAMP sensor (KcAMP $1 / 43 \mathrm{mM}$ ). We monitored the activity of the CNG channel by its $\mathrm{Ca}^{2+}$ permeability using the fluorescent $\mathrm{Ca}^{2+}$ indicator Fluo-4. We used the same excitation light (lexc $1 / 4480 \pm 10 \mathrm{~nm}$ ) to stimulate PACa and Fluo-4. When we increased the light intensity tenfold, control cells only displayed a step increase of Fluo-4 fluorescence (Fig. 2a), whereas PACaexpressing cells displayed an additional slower increase in fluorescence, indicating lightstimulated $\mathrm{Ca}^{2+}$ influx through CNG channels (Fig. 2b). To monitor $\mathrm{Ca}^{2+}$ without strongly stimulating PACa, we used a photoshutter to produce short flashes of light to measure Fluo-4 fluorescence. The $\mathrm{Ca}^{2+}$ signal reached a plateau upon continuous light stimulation; when we closed the photoshutter and probed the fluorescence every $2 \mathrm{~s}$ with a short flash of light, the $\mathrm{Ca}^{2+}$ signal declined to a baseline level within about $30 \mathrm{~s}$, reflecting phosphodiesterase activity of the cell (Fig. 2c). In the presence of IBMX (100 mM), the lightstimulated $\mathrm{Ca}^{2+}$ response was similar, but the signal declined only slightly. Finally, we investigated the resting activity of PACa by monitoring the fluorescence of Fluo-4 without continuous illumination (Fig. 2d). Upon addition of IBMX to the bath, the fluorescence increased slowly and reached a stable plateau after B200 $\mathrm{s}$. We observed no such increase in control cells that expressed the CNGA2-T537M channel only (data not shown).

To test the potential of transgenic PAC expression in vivo,we used the Gal4UAS enhancer expression system ${ }^{14}$ for targeting of PACs to the D. melanogaster brain. Neuronal expression of either PACa or PACb with the elav-Gal4 driver line resulted in adult flies without any obvious behavioral phenotype. Irradiating adult flies with blue light resulted in periods of hyperactivity and unusual freezing behavior in flies expressing PACa (Supplementary Video 1 online), but not in wild-type flies (Supplementary Video 2 online) or in flies expressing PACb, even under the control of the 
strong tubulin promoter (tub-Gal4/UAS-PACb; Supplementary

[Begin of page 41]

Fig. 3 online). Similarly, irradiation of elav-Gal4-UAS-PACa flies with the same light intensity but different lexc $(590 \pm 10 \mathrm{~nm})$ did not affect behavior (Supplementary Video 3 online).

To determine the reliance and kinetics of the light-induced change in behavior, we analyzed the grooming reflex ${ }^{15}$. When covered with a fine powder, fruit flies instantaneously display vigorous and continuous grooming activity lasting up to $30 \mathrm{~min}$ (data not shown). Monitoring this behavior for a total time of 5 min with irradiation alternating between dim white light (from a cold light source) and intense blue light (from a light-emitting diode, lexc 1/4 $455 \pm 10 \mathrm{~nm}$ ) for $1 \mathrm{~min}$ each, revealed high grooming activity in wild-type Canton-S flies irrespective of stimulation by light (Fig. 3). In contrast, neuronal expression of PACa (elav-Gal4/ UAS-PACa) resulted in hyperactivity and a substantial decline in grooming activity under blue-light stimulation. When irradiation was switched back to dim white light, thus turning off blue light- induced PAC activity, flies returned to grooming behavior within several seconds (Supplementary Video 1 and Supplementary Table 1 online). These results demonstrate that transgenic expression of PACa in fruit flies results in a functional protein that is rapidly and reversibly activated by blue light. Moreover, the fast action observed at the on- and offset of irradiation demonstrates the feasibility of rapid control of cAMP levels in a freely moving animal.

In this study, we have successfully expressed the PACa and PACb from the flagellate E. gracilis in multiple systems where they functioned as lightsensitive adenylyl cyclases, albeit with different enzymatic activities. We conclude that enzymatic turnover, that is, light-activated cAMP production, of PACa is about 100-fold that of PACb. Notably, we demonstrate that the free cAMP concentration produced by light stimulation is sufficient to activate important targets for cAMP, namely cAMPdependent protein kinase (PKA) and CNG channels. This tool may provide exquisite spatiotemporal control of cAMP levels in future work of signaling pathways in transgenic models, particularly for tackling questions of learning and memory in Drosophila. Advantages of this new method are: (i) the adenylyl cyclase is genetically encoded by a single gene; (ii) no chemical modification of the protein is required; (iii) no addition of chromophore is needed; (iv) the substrate (ATP) is plentiful; and (v) the chromophore or expressed protein is not toxic if expression is limited.

[Begin of page 42 ]

Compared to photo-uncaging, this method is advantageous as no precursor is exhausted, it is easier to use, and it faces no complications with diffusion or premature degradation of the caged compound.

The basal activity of PACa may be a disadvantage for specific applications, but there are alternatives: weak expression of PACa or expression of the 100-fold less active PACb. Future work on PACs might allow suppression of resting activity by appropriate mutation(s), thereby further improving a powerful tool 
for manipulating [cAMP] by light.

\section{ACKNOWLEDGMENTS}

We are indebted to J.W. Karpen (Oregon Health \& Science University) for a generous gift of the CNGA2-C46oW,E583M plasmid. S.S.-L. and G.N. thank E. Bamberg (Max-Planck-Institut für Biophysik) for generous support. D. Ollig,

M. Dörr, T. Schiereis, A. Jaeckel, M. Glander and J. Wiegant provided excellent technical support. Members of the Drosophila Genomic Resource Center (Bloomington University) provided us with T. Murphey's GATEWAY collection of expression vectors. The work was supported by the Deutsche Forschungsgemeinschaft (to P.H. and G.N.) and the Max-Planck-Gesellschaft (to G.N.).

1. Cooper, D.M. Biochem. J. 375, 517-529 (2003).

2. Iseki, M. et al. Nature 415, 1047-1051 (2002).

3. Anderson, S. et al. Biochemistry 44, 7998-8005 (2005).

4. Gauden, M. et al. Proc. Natl. Acad. Sci. USA 103, 10895-10900 (2006).

5. Nagel, G. et al. Curr. Biol. 15, 2279-2284 (2005).

6. Schroll, C. et al. Curr. Biol. 16, 1741-1747 (2006).

7. Zhang, F., Wang, L.-P., Boyden, E.S. \& Deisseroth, K. Nat. Methods 3, 785792 (2006).

8. Riordan, J.R. et al. Science 245, 1066-1073 (1989).

9. Nagel, G. et al. Nature 360, 81-84 (1992).

10. Weinreich, F., Wood, P.G., Riordan, J.R. \& Nagel, G. Pflugers Arch. 434, 484-491 (1997).

11. Altenhofen, W. et al. Proc. Natl. Acad. Sci. USA 88, 9868-9872 (1991).

12. Rich, T.C. et al. Proc. Natl. Acad. Sci. USA 98, 13049-13054 (2001).

13. Yoshikawa, S., Suzuki, T., Watanabe, M. \& Iseki, M. Photochem. Photobiol. Sci. 4, 727-731 (2005).

14. Brand, A.H. \& Perrimon, N. Development 118, 401-415 (1993).

15. Melzig, J. et al. J. Neurosci. 18, 7160-7166 (1998). 